\title{
Rhabdomyolysis Triggered by an Asthmatic Attack in a Patient with McArdle Disease
}

\author{
Kenji Tsushima, Shigeru Koyama*, Mayumi Ueno**, Keisaku Fujimoto, Takeo Ichiyoshi*, \\ Yo-ichi TAKEI ${ }^{* * *}$, Norinao HANYU*** and Keishi KuBo
}

\begin{abstract}
We describe a patient with McArdle disease who developed rhabdomyolysis triggered by a bronchial asthmatic attack. A 64-year-old man had chronic pulmonary emphysema with asthma, and an asthmatic attack led to severe rhabdomyolysis that required continuous hemodiafiltration. After 2 years, a physical examination revealed atrophy of the extremities compared with previous examinations, especially of the intercostal muscles. During that time, he suffered two severe bronchial asthmatic attacks. His serum level of creatinine kinase remained between 4,000 and 7,000 IU/l when he did not suffer from asthmatic attacks and rhabdomyolysis had abated. Therefore, we suspected that his recent muscle atrophy was caused by asthmatic attacks, and discussed the possibility of his respiratory muscle weakness due to McArdle disease in relation to his severe bronchial asthmatic attacks as well as chronic obstructive pulmonary disease.
\end{abstract}

(Internal Medicine 40: 131-134, 2001)

Key words: creatinine kinase, pulmonary emphysema, respiratory muscle's atrophy

\section{Introduction}

Phosphorylase plays a major role in glycolysis. There are three mammalian isozymes (muscle, liver and brain type), and genetic defect of the muscle-specific isozyme (myophosphorylase) causes a metabolic myopathy known as glycogenosis type $\mathrm{V}$, or McArdle disease (1). In patients with myophosphorylase deficiency, exercise intolerance usually occurs in childhood, even though in most patients the diagnosis is not made until their second or third decade of life. In the present patient, rhabdomyolysis was triggered by an attack of bronchial asthma and led us to diagnosis of McArdle disease. We describe his clinical course after rhabdomyolysis and have evaluated atrophy of the respiratory muscles retrospectively.

\section{Case Report}

In 1988 a 58-year-old man who had smoked 20 cigarettes a day for 45 years visited the Nagano Red Cross Hospital with dyspnea, and the chest $\mathrm{X}$-ray finding and pulmonary function tests were compatible with pulmonary emphysema. He had cramps on exercise. In January 1996 dyspnea on effort gradually became more severe, and he underwent home oxygen therapy. On February 6, 1996, he was wheezing, had dyspnea, complained of general fatigue, and was admitted to the Nagano Red Cross Hospital. He did not take drugs that could potentially cause myopathy. His family was not consanguineous. His pulse rate was 117 per minute and his blood pressure was 140/ $90 \mathrm{mmHg}$. He presented orthopnea, cyanosis and wheezing, however, his heart rhythm was normal. Neurological findings revealed a slight weakness of the bilateral upper arms, but did not reveal an apparent atrophy of intracostal muscle. Hematological and biochemical analyses revealed the following: white blood cells, $9,600 / \mathrm{mm}^{3}$ without hypereosinophilia; c-reactive protein, $6.0 \mathrm{mg} / \mathrm{dl}$ (normal, $<0.2 \mathrm{mg} / \mathrm{dl}$ ); lactate dehydrogenase, 3,513 IU/l (normal, 150 to $400 \mathrm{IU} /$ ) ); aspartate aminotransferase, $588 \mathrm{IU} / l$ (normal, 5 to $35 \mathrm{IU} / l$ ); creatinine kinase (CK), 50,932 IU/l (normal, 24 to $195 \mathrm{IU} / l$ ); blood urea nitrogen, 15.0 $\mathrm{mg} / \mathrm{dl}$ (normal, 5 to $20 \mathrm{mg} / \mathrm{dl}$ ), and creatinine, $1.3 \mathrm{mg} / \mathrm{dl}$ (nor$\mathrm{mal}, 0.4$ to $1.1 \mathrm{mg} / \mathrm{dl}$ ). Urinalysis showed 8 to 10 erythrocytes and a $3+$ reaction for blood on the dipstick. Thus, he was diagnosed as having rhabdomyolysis based on his high level of serum creatinine kinase. Arterial blood gas analysis in room air revealed $50.3 \mathrm{mmHg}$ of $\mathrm{PaO}_{2}, 32.4 \mathrm{mmHg}$ of $\mathrm{PaCO}_{2}, 28.5 \mathrm{mM} /$ $l$ of $\mathrm{HCO}_{3}{ }^{-}$and $\mathrm{pH}$ 7.40. The chest X-ray and computed tomographic scan of the chest revealed moderate emphysema. He was diagnosed to have emphysema with asthma. Prednisolone (20 mg/day) and bronchodilator (aminophilin; $500 \mathrm{mg} /$ day)

From the First Department of Internal Medicine, Shinshu University School of Medicine, Matsumoto, *the Department of Internal Medicine, Nagano Red Cross Hospital, Nagano, **the Second Department of Internal Medicine, Shinshu University School of Medicine, Matsumoto and ***the Department of Neurology, Nagano Red Cross Hospital, Nagano

Received for publication February 3, 2000; Accepted for publication August 14, 2000

Reprint requests should be addressed to Dr. Kenji Tsushima, the First Department of Internal Medicine, Shinshu University School of Medicine, 3-1-1 Asahi, Matsumoto 390-8621 
were administered by intravenous drip infusion, and hemodiafiltration was attempted for rhabdomyolysis. On the next day, CK had increased to $120,980 \mathrm{IU} / \mathrm{l}$ and the patient was anuric, and, thus, continuous hemodiafiltration was given. We also suspected a form of glycogen storage disease because of the high CK values and the existence of muscle cramps in his past history. The semi-ischemic forearm exercise test was performed for suspected glycogen storage disease. No electrophysiological examinations were carried out because we could not obtain the patient's consent. A biceps muscle biopsy specimen

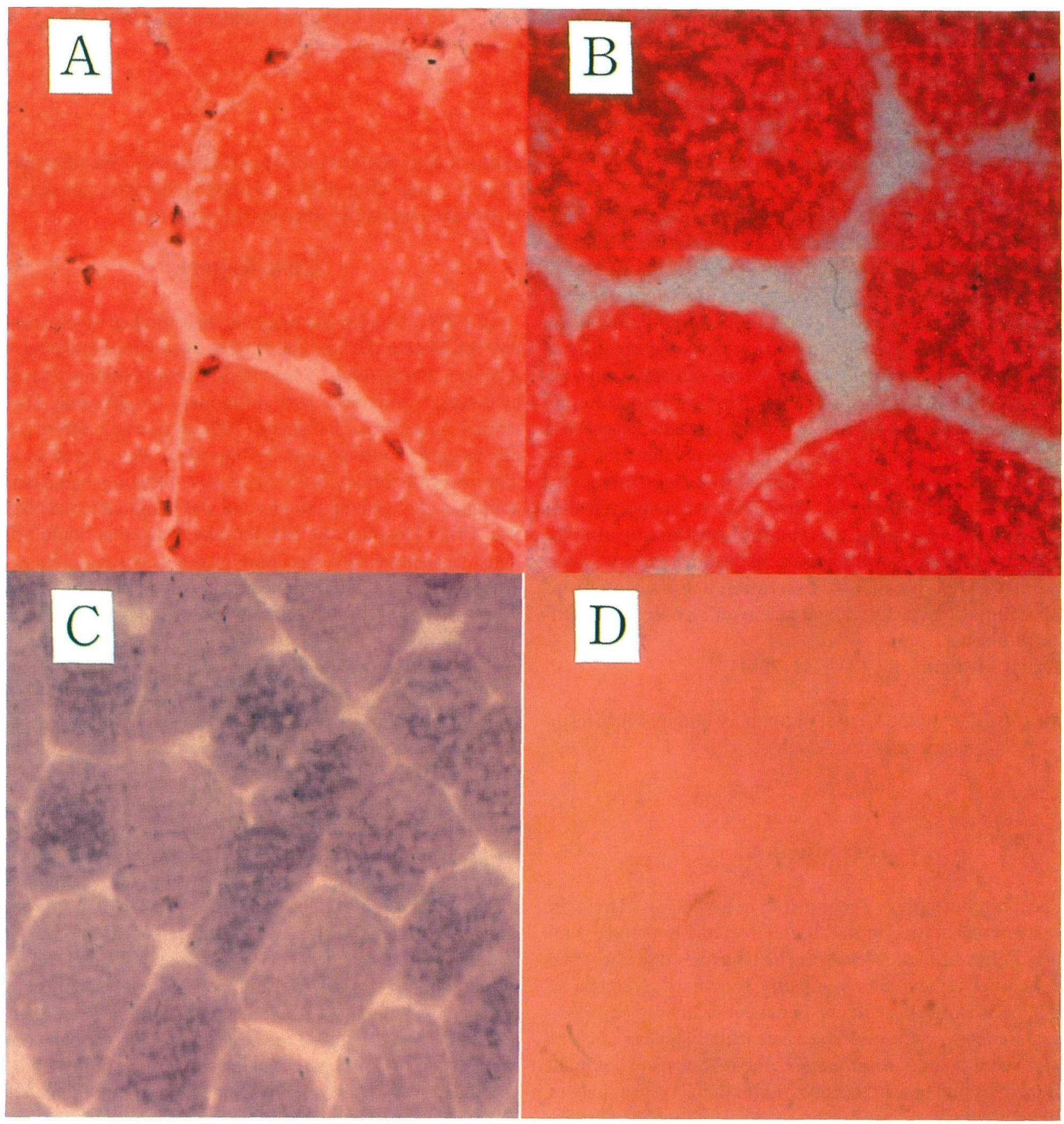

Figure 1. Specimen obtained from the right upper biceps brachii muscle biopsy. A) hematoxylin-eosin staining shows no abnormalities $(\times 400)$. B) Periodic acid Schiff staining shows an increased amount of subsarcolemmic glycogen $(\times 400)$. C) Autopsy specimens obtained from the right upper biceps brachii muscle biopsy. The histochemical staining for myophosphorylase is positive $(\times 200)$. D) The histochemical staining for myophosphorylase is negative. These photos show tiny clear spots caused by ice crystal artifacts and not microvacuolations containing glycogen granules. 
revealed no abnormalities on hematoxylin and eosin preparations, but periodic Schiff staining revealed an increased amount of subsarcolemmic glycogen in several fibers, which was completely digested by diastase (Fig. 1). Cytochrome c oxidase showed a positive staining, however, myophosphorylase was negative. Therefore, the patient was diagnosed to have McArdle disease associated with pulmonary emphysema and bronchial asthma. During the following two-year period he had two episodes of rhabdomyolysis associated with asthmatic attacks, but inhalation of beclomethasone dipropionate has prevented the occurrence of rhabdomyolysis, thereafter. His serum level of CK remained elevated between 4,000 and 7,000 IU/l, even when he had no evidence of asthmatic attacks or rhabdomyolysis.

\section{Discussion}

The cardinal symptom of myophosphorylase deficiency is exercise intolerance manifested by myalgia, early fatigue and stiffness or the weakness of exercising muscles, and relief of these symptoms at rest (2). The exercise intolerance typically starts in childhood, but the diagnosis is often delayed. Muscle necrosis associated with myoglobinuria occurs in about half of all patients with myophosphorylase deficiency, invariably after strenuous or prolonged exercise and usually proceeded by myalgia, muscle cramps or both (3). In the present patient, asthmatic attack exposed the respiratory muscles to strenuous and prolonged exercise. The skeletal muscles are supplied with energy derived from fatty acid catabolism at rest and during mild exercise. However, when the oxygen supply is insufficient due to aerobiosis, heavy exercises are supplied by the process of glycogenolysis. Since McArdle disease is a glycogenolysis disorder, the patient deteriorated quickly in the face of poor oxygenation complicated with bronchial asthma. This further hampered the function of the respiratory muscles and circulatory systems (4).

In the present patient, atrophy of the intercostal muscles and upper arm muscles became prominent within 2 years. The main clue for diagnosis was the result of the past pulmonary func- tion tests (Table 1). Diseases associated with progressive muscle atrophy include amyotrophic lateral sclerosis, muscular dystrophy and inflammatory myopathy. The natural history of McArdle disease is usually benign apart from exercise-induced cramps and occasional rhabdomyolysis. In those patients, however, their vital capacity drops more than $50 \%$ of the predicted value (5). Generally, the vital capacity, maximal voluntary ventilation and forced expiratory volume do not drop significantly until respiratory muscle function is compromised. In many cases the residual volume is within the normal range, but the functional residual capacity drops. Rahn et al established the relationship between lung inflation and maximal static respiratory pressures (6). It is near functional residual capacity that all inspiratory muscles combined are at their optimal length and provide the highest intrathracic inspiratory force. The sensitive useful diagnostic test for the respiratory muscle strength is the maximal inspiratory and expiratory pressures. Values in patients with chronic obstructive pulmonary disease have been reported (7). Although the pulmonary emphysema was not profound, those parameters were significantly worse; his maximal inspiratory pressure was $34 \mathrm{cmH}_{2} \mathrm{O}$, his maximal expiratory pressure was $48 \mathrm{cmH}_{2} \mathrm{O}$ and his maximal voluntary ventilation was $22 \mathrm{cmH}_{2} \mathrm{O}(8)$.

McArdle disease complicated with respiratory disease is rare. Therefore, it is unknown whether McArdle disease induces weakness of respiratory muscle. Faigel reported that type II carnitine palmitoyltransferase deficiency, which has similar symptoms to McArdle disease has shown proximal muscle weakness (9). It is suspected that the severe attack of bronchial asthma complicated with McArdle disease induced muscle injury and weakness in the present patient.

In the present patient, who had McArdle disease complicated with bronchial asthma, energy demands, supply and storage were all problematic. The more asthmatic attacks he might suffer in the future, the greater the atrophy of the respiratory muscles. We must, therefore, carefully monitor this patient to prevent further progression of respiratory weakness. To avoid asthmatic attacks would be the key to maintain his respiratory

Table 1. Pulmonary Function Test

\begin{tabular}{|c|c|c|c|c|c|c|c|}
\hline \multirow[b]{2}{*}{$\% \mathrm{VC}(\%)$} & \multirow[b]{2}{*}[\mathrm{VC}(l)]{} & \multicolumn{2}{|c|}{$3 / 23 / 1992$} & \multicolumn{2}{|c|}{$7 / 14 / 1995$} & \multicolumn{2}{|c|}{$6 / 18 / 1997$} \\
\hline & & 61.0 & {$[2.10]$} & 66.0 & {$[2.25]$} & 66.2 & [2.17] \\
\hline $\mathrm{FEV}_{1.0} \%(\%)$ & {$\left[\mathrm{FEV}_{1.0}(l)\right]$} & 52.3 & [1.18] & 46.0 & {$[0.97]$} & 48.5 & [0.97] \\
\hline$\% \mathrm{TLC}(\%)$ & {$[\operatorname{TLC}(l)]$} & 89.2 & [4.80] & \multicolumn{2}{|c|}{ ND } & 90.3 & {$[4.75]$} \\
\hline$\% \mathrm{RV}(\%)$ & {$[\mathrm{RV}(l)]$} & 148 & {$[2.42]$} & \multicolumn{2}{|c|}{ ND } & 134 & [2.27] \\
\hline \multicolumn{2}{|c|}{$\mathrm{DLco}(\mathrm{ml} / \mathrm{min} / \mathrm{mmHg})$} & \multicolumn{2}{|c|}{13.7} & \multicolumn{2}{|c|}{13.0} & \multicolumn{2}{|c|}{10.7} \\
\hline \multicolumn{2}{|c|}{$\operatorname{MVV}(l / \min )$} & \multicolumn{2}{|c|}{ ND } & \multicolumn{2}{|c|}{24.0} & \multicolumn{2}{|c|}{24.3} \\
\hline \multicolumn{2}{|c|}{$\mathrm{MIP}\left(\mathrm{cmH}_{2} \mathrm{O}\right)$} & \multicolumn{2}{|c|}{ ND } & \multicolumn{2}{|c|}{-57.4} & \multicolumn{2}{|c|}{-33.2} \\
\hline \multicolumn{2}{|c|}{$\operatorname{MEP}\left(\mathrm{cmH}_{2} \mathrm{O}\right)$} & \multicolumn{2}{|c|}{ ND } & \multicolumn{2}{|c|}{74.2} & \multicolumn{2}{|c|}{48.0} \\
\hline
\end{tabular}

\%VC: \% predicted vital capacity, $\mathrm{FEV}_{1.0 \%}: \%$ forced expiratory volume in 1 second, \% TLC: \% predicted total lung capacity, \%RV: \% predicted residual volume, DLco: diffusing capacity for carbon monoxide, MVV: maximal voluntary ventilation, MIP: maximal inspiratory pressure, MEP: maximal expiratory pressure. ND: not done. 


\section{Tsushima et al}

function.

\section{References}

1) McArdle B. Myopathy due to a defect in muscle glycogen breakdown. Clin Sci 10: 13-33, 1951.

2) Mommaerts WFHM, Illingworth B, Pearson CM, Guillory R, Seraydarian $\mathrm{K}$. A functional disorder of muscle associated with the absence of phosphorylase. Proc Natl Acad Sci USA 45: 791-797, 1959.

3) Kristjansson K, Tsujino S, DiMauro S. Myophosphorylase deficiency: An unusually severe form with myoglobinuria. J Pediatr 125: 409-410, 1994.

4) Pryor SL, Lewis SF, Haller RG, Bertocci LA, Victor RG. Impairment of sympathetic activation during static exercise in patients with muscle phosphorylase deficiency (McArdle's disease). J Clin Invest 85: 1444-1449, 1990.

5) Irvin CG. Lung volume. Semin Respir Crit Care Med 19: 325-334, 1998.

6) Rahn H, Otis A, Chadwick L, et al. The pressure volume diagram of the thorax and lung. Am J Physiol 146: 161-173, 1946.

7) Rochester DF, Arora NS. Respiratory muscle failure. Med Clin North Am 67: 573-597, 1983.

8) American Thracic Society. Lung function testing: section of reference values and interpretative strategies (statement). Am Rev Respir Dis 144: 1201-1218, 1991.

9) Faigel HC. Carnitine palmitoyltransferase deficiency in a college athlete: a case report and literature review. J Am Coll Health 44: 51-54, 1995. 